\title{
FBXL16 is a novel E2F1-regulated gene commonly upregulated in p16INK4A- and p14ARF-silenced HeLa cells
}

\author{
KAZUYUKI SATO, YUSUKE KUSAMA, MOE TATEGU and KENICHI YOSHIDA \\ Department of Life Sciences, Faculty of Agriculture, Meiji University, \\ 1-1-1 Higashimita, Tama-ku, Kawasaki, Kanagawa 214-8571, Japan
}

Received August 28, 2009; Accepted October 16, 2009

DOI: 10.3892/ijo_00000522

\begin{abstract}
Two crucial cell cycle regulators, p16INK4A and p14ARF, are produced from the cyclin-dependent kinase inhibitor $2 \mathrm{~A}(C D K N 2 \mathrm{~A})$ gene locus by alternative reading frames; these regulators act as tumor suppressors during tumorigenesis. However, the molecular events incidental to the acute functional loss of $C D K N 2 A$ remain a critical issue. Two pivotal regulatory pathways of cell fate determination involving p16INK4A/retinoblastoma protein (pRb)/E2F1 and p14ARF/p53 interact tightly with each other; however, novel factors with an integral or overlapping role in these two pathways remain incompletely defined. To this end, we specifically decreased the expression of p16INK4A or p14ARF proteins using RNA interference (RNAi) in HeLa cells. Using a DNA microarray approach, we showed that several genes are commonly regulated in both p16INK4A and p14ARF knockdown cells, compared with control RNAtreated cells. We focused on the FBXL16 (F-box and leucinerich repeat protein 16) gene, the expression of which was reproducibly upregulated in p16INK4A and p14ARF knockdown cells, as evaluated using RT-PCR. Interestingly, the promoter region of FBXL16 was shown to be upregulated by activator E2Fs. Finally, RNAi-mediated knockdown of FBXL16 increased the cell proliferation rate of HeLa cells. Together, our results illustrate a unique aspect of the interdependence between the p16INK4A/pRb/E2F1 and p14ARF/ p53 pathways at a molecular level.
\end{abstract}

Correspondence to: Dr Kenichi Yoshida, Department of Life Sciences, Faculty of Agriculture, Meiji University, 1-1-1 Higashimita, Tama-ku, Kawasaki, Kanagawa 214-8571, Japan

E-mail: yoshida@isc.meiji.ac.jp

Abrreviations: CDKN2A, cyclin-dependent kinase inhibitor 2A; DMSO, dimethyl sulfoxide; FBXL16, F-box and leucine-rich repeat protein 16; GAPDH, glyceraldehyde-3-phosphate dehydrogenase; $\mathrm{PCR}$, polymerase chain reaction; $\mathrm{pRb}$, retinoblastoma protein; RLU, relative light units; RNAi, RNA interference; RT, reverse transcription; siRNA, small interference RNA

Key words: FBXL16, E2F1, p16INK4A, p14ARF, gene silencing

\section{Introduction}

The cyclin-dependent kinase inhibitor $2 \mathrm{~A}(C D K N 2 A)$ gene at human chromosome 9p21 encodes two proteins, p16INK4A and $\mathrm{p} 14 \mathrm{ARF}$, that are produced by alternative reading frames, and the INK4/ARF locus, including p15INK4B $(C D K N 2 B)$, is frequently deleted in many human cancers $(1,2)$. These proteins are uniquely involved in the regulation of cell cycle and apoptosis. Namely, p16INK4A antagonizes CDK4 and CDK6 activities to maintain retinoblastoma protein (pRb) in its active hypophosphorylated state and to suppress E2F1 transcriptional activity (as well as the activities of other activator E2Fs), while p14ARF binds and suppresses the oncoprotein MDM2 (mouse double minute 2 homolog), leading to the stabilization of tumor suppressor p53. Thus, these two pivotal cell fate regulatory pathways, p16INK4A/ $\mathrm{pRb} / \mathrm{E} 2 \mathrm{~F} 1$ and $\mathrm{p} 14 \mathrm{ARF} / \mathrm{p} 53$, are known to be responsible for more than half of all instances of human tumorigenesis $(3,4)$. Moreover, these two pathways are mutually linked at various levels. For example, p14ARF is a transcriptional target of E2F1, and p14ARF conversely inhibits E2F activity (5,6). A well-known p53 target, CDKN1A (p21CIP1/WAF1), is also regulated by $\mathrm{E} 2 \mathrm{~F} 1$, and $\mathrm{p} 21 \mathrm{CIP} 1 / \mathrm{WAF} 1$ negatively regulates E2F1 activity by preventing the CDK-dependent phosphorylation of $\mathrm{pRb}$ or by direct interaction with E2F1 (7-9). A functional interaction between $\mathrm{pRb} / \mathrm{E} 2 \mathrm{~F} 1$ and MDM2 has also been reported (10).

Based on experimental evidence that the double knockout of both pl6Ink4a and p19Arf (a mouse counterpart of human $p 14 A R F)$ in mice resulted in a severe cancer-prone phenotype, compared with the specific knockout of either p16Ink4a or p19Arf in mice, these two regulatory pathways might be even more tightly interdependent than previously imagined. Several critical questions remain to be answered. First, which protein, p16INK4A or p14ARF, is responsible for tumorigenesis in humans remains incompletely answered, though the loss of p19Arf had a greater impact on tumorigenesis than the loss of p16Ink $4 a$ in a mouse model $(11,12)$. Second, tumorigenesis occurs over a long period of time, especially in humans; therefore, the types of molecular events that occur when p16INK4A and p14ARF are functionally inactivated remain uncertain. This kind of real-time investigation cannot be performed in human clinical cases but can be accomplished using RNA interference (RNAi) technology and human cultured cells, although conditional knockout mice are 
another option. Apparently, the functional loss of p16INK4A and p14ARF eventually disrupts the cell cycle and the control of apoptosis, but the initial primary events induced by the loss of these two proteins remain unclear in human somatic cells. We hypothesized that if the initial molecular events incidental to the acute loss of p16INK4A and p14ARF could be reproduced and analyzed, a novel paradigm for at least identifying molecular targets that are potentially located downstream of the p16INK4A/pRb/E2F1 and p14ARF/p53 pathways might be established.

In this study, we first specifically decreased the protein level of either p16INK4A or p14ARF in HeLa cells and then analyzed the global changes in gene expression in p16INK4A or p14ARF knockdown cells. Surprisingly, several genes that were regulated similarly in the p16INK4A and the p14ARF knockdown cells were identified. Uniquely, FBXL16, which was commonly upregulated in p16INK4A and p14ARF knockdown cells, was identified as a novel transcriptional target of activator E2Fs, and functional loss of FBXL16 increased the cell proliferation rate of HeLa cells. Our present data might suggest a unique platform for elucidating the underlying molecular mechanism shared by the $\mathrm{p} 16 \mathrm{INK} 4 \mathrm{~A} / \mathrm{pRb} / \mathrm{E} 2 \mathrm{~F}$ and $\mathrm{p} 14 \mathrm{ARF} / \mathrm{p} 53$ pathways.

\section{Materials and methods}

Cell culture, small interference RNA (siRNA) transfection, and chemical. WI-38 (human embryonic fibroblast), A549 (lung adenocarcinoma), and HeLa (cervical carcinoma) cells were cultured in Earle's modified Eagle's medium (MEM) (Invitrogen, Carlsbad, CA) supplemented with 10\% fetal bovine serum (FBS), $1 \%$ non-essential amino acids (Invitrogen), and antibiotic-antimycotics (Invitrogen).

The Stealth RNA for silencing of p16INK4A (240 and 319), p14ARF (169 and 279), FBXL16 (HSS135226) and negative control high GC duplex were purchased from Invitrogen. The RNA sequences of sense and antisense orientations were as follows; 240 (UGGCCAGCCAGUCAGCC GAAGGCUC and GAGCCUUCGGCUGACUGGCUGG CCA), 319 (UAACUAUUCGGUGCGUUGGGCAGCG and CGCUGCCCAACGCACCGAAUAGUUA), 169 (AAUCCG GAGGGUCACCAAGAACCUG and CAGGUUCUUGGUG ACCCUCCGGAUU), 279 (AUCAGCACGAGGGCCACAG CGGCGG and CCGCCGCUGUGGCCCUCGUGCUGAU) and HSS135226 (CCGAGCUCUUCAAGUAUUUCUCGCA and UGCGAGAAAUACUUGAAGAGCUCGG). Transfection was performed using the Lipofectamine 2000 reagent (Invitrogen), according to the manufacturer's instructions. Cells were plated into $60-\mathrm{mm}$ dishes $24 \mathrm{~h}$ prior to transfection. At the time of transfection, the cell density was $1-5 \times 10^{5}$ cells $/ \mathrm{ml}$. Briefly, 125 pmol of siRNA and $2.5 \mu 1$ of transfection reagent were incubated in $0.5 \mathrm{ml}$ of Opti-MEM I Reduced Serum Medium (Invitrogen) for 15 min to facilitate complex formation at room temperature. The resulting mixture was added to the cells cultured in $1.5 \mathrm{ml}$ of MEM (day 0). After $24 \mathrm{~h}$, siRNA/transfection reagent mixture was re-added (day 1).

Etoposide was purchased from Wako Pure Chemical Industries (Osaka, Japan) and dissolved in dimethyl sulfoxide (DMSO) as $100 \mathrm{mM}$ stock solutions and kept at $-20^{\circ} \mathrm{C}$ until dilution before use.
Plasmid construction. Promoter fragments were generated with the polymerase chain reaction (PCR) method from human genomic DNA (Promega, Madison, WI), and ligated into the respective enzyme $K p n I$ and $B g l$ II sites of pGL3Basic vector (Promega). PCR primers were designed on public genome sequence (GenBank No. AE006464) as follows (underlines indicate flanking enzyme site); -646, GGGGTAC CCTCGGGTGCTTTCACTGTCA; -579, GGGGTACCGTG CCAGGGTCTCCAATGAG; -306, GGGGTACCTTCTGCC TCCCGCGCCCTCC; -287, GAAGATCTGGAGGGCGCG GGAGGCAGAA; -90, GAAGATCTGTGGCGAGCCAATC CGGGTT; +44, GAAGATCTAAGGCCGCTTTGCAGGG $\mathrm{AAC}$, where minus position is relative to transcriptional start site as +1 . All the plasmid sequences were verified by sequencing at the Takara facility (Mie, Japan).

For the construction of N-terminally FLAG-tagged FBXL16 expression plasmid, coding region of FBXL16 was amplified by PCR primers (CGGAATTCGAGCCCGGGCA TCGACGGC and CGGGATCCCTACTCAATGACGAGG CAGCG) (underlines indicate EcoRI and BamHI restriction enzyme sites) and FBXL16 EST clone (GenBank No. BC036680; IMAGE 5262152, Open Biosystems, Huntsville, $\mathrm{AL})$ as a template. Amplified fragments were ligated into p3xFLAG-CMV-10 (Sigma, Saint Louis, MO). pcDNA3E2F1 (Dr J.R. Nevins, Duke University Medical Center, USA), pRK5-V12 HRAS (Dr A. Tolkovsky, University of Cambridge, UK), pF-Bmi (Dr J.L. Hess, University of Pennsylvania Medical Center) and pcGN-HA-CDC6 (Dr S. Gonzalez and Dr M. Serrano, CNIO, Madrid) were kindly provided. pcDNA3 was purchased from Invitrogen.

Reverse transcription (RT)-PCR. On day 2 of siRNA transfection protocol, total RNA samples were prepared using RNeasy mini-spin column (Qiagen, Valencia, CA), in accordance with the manufacturer's instructions. Total RNA $(2 \mu \mathrm{g})$ was reverse transcribed using High-Capacity cDNA Reverse Transcription kits (Applied Biosystems, Foster City, CA). The PCR was carried out in $25 \mu 1$ of a mix consisting of $1 \mathrm{X}$ buffer, $200 \mu \mathrm{M}$ dNTPs, $400 \mathrm{nM}$ primers, $1 \mathrm{mM}$ $\mathrm{MgSO}_{4}, 5 \%$ DMSO and 1 unit of KOD plus DNA polymerase (Toyobo, Osaka, Japan). Hot-start PCR was then performed as follows: denaturation for $3 \mathrm{~min}$ at $94^{\circ} \mathrm{C}$, followed by ad libitum cycles at $94^{\circ} \mathrm{C}$ for $15 \mathrm{sec}$, gene-specific annealing temperature $\left(F B X L 16,55^{\circ} \mathrm{C} ; \operatorname{EIF} 4 E B P 2,54^{\circ} \mathrm{C}\right.$; $\left.A R M C X 6,54^{\circ} \mathrm{C} ; G A P D H, 60^{\circ} \mathrm{C}\right)$ for $30 \mathrm{sec}$, and $68^{\circ} \mathrm{C}$ for $30 \mathrm{sec}$, and the last one was followed by an extension step of $3 \mathrm{~min}$ at $68^{\circ} \mathrm{C}$. The PCR results were verified by varying the number of PCR cycles for each cDNA and set of primers. The target gene primer pairs were as follows; FBXL16 (NM_153350), TCAGGCCTGGGAAGAGGCCT and AGGGAGTGTCCCAAGATTGG for 440-bp; EIF4EBP2 (NM_004096), GCACAGCTTGTGCAACTCTG and CAGGCCTTACCCACCAAGTG for 340-bp; ARMCX6 (NM_019007), GGGCTCAATCCAGGACCACA and GTTCACTATCCATCAGGCGC for 350-bp; GAPDH (NM_002046), CCATGGCAAATTCCATGGCA and GTCCTTCCACGATACCAAAG for 365-bp. The amplified products were separated on $1.0-1.5 \%$ agarose gels and visualized under ultraviolet transillumination. For cDNA panel analysis, $2.5 \mu \mathrm{l}$ of cDNA purchased from Clontech 
(Mountain View, CA) was used (Human MTC panel I and II, and Fetal MTC panel). GAPDH $(G 3 P D H)$ primer in the kit was used as a control to amplify 983-bp.

Western blot. Cells were transfected with Lipofectamine Plus (Invitrogen) according to manufacturer's instruction. Briefly, $1-3 \times 10^{5}$ cells were inoculated into 6-well plate the day before transfection. Plasmid DNA (0.5-2 $\mu \mathrm{g}$ for each well) mixed with Lipofectamine Plus reagent was directly added to the cell culture. After indicated time period, cells were harvested and lysed in RIPA lysis buffer $(50 \mathrm{mM}$ Tris- $\mathrm{HCl}, \mathrm{pH} 7.6$, $150 \mathrm{mM} \mathrm{NaCl}, 1 \%$ Nonidet $\mathrm{P} 40,0.5 \%$ sodium deoxycholate, $0.1 \%$ SDS, $1 \mathrm{mM}$ EDTA, $1 \mathrm{mM}$ PMSF, and $1 \mu \mathrm{g} / \mathrm{ml}$ each of aprotinin, pepstatin and leupeptin) for $20 \mathrm{~min}$ on ice. The cell lysates were centrifuged and the protein concentration was determined using the Bio-Rad protein assay kit (Bio-Rad laboratories, Hercules, CA). Before being subjected to SDSpolyacrylamide gel electrophoresis (PAGE), the reaction was stopped by the addition of LDS sample buffer (Invitrogen) containing $100 \mathrm{mM}$ DTT. After being heated at $70^{\circ} \mathrm{C}$ for $10 \mathrm{~min}$, equal amounts of cellular protein $(40 \mu \mathrm{g}$ otherwise indicated) were electrophoresed on NuPAGE 4-12\% Bis-Tris gel with MES running buffer (Invitrogen), and transferred to a Hybond ECL nitrocellulose membrane (GE Healthcare, Piscataway, NJ). The membrane was first blocked in phosphatebuffered saline (PBS) containing $0.1 \%$ Tween-20 and 5\% non-fat dried milk and then incubated with the following antibodies: p16INK4A (sc-467), p15INK4B (sc-612), p53 (sc-6243), p53 Ser15 (sc-11764), p21WAF1/CIP1 (sc-397), MDM2 (sc-965), E2F1 (sc-193), cyclin A (sc-239), cyclin E (sc-198), and HRAS (sc-520) were purchased from Santa Cruz Biotechnology (Santa Cruz, CA). Anti-p14ARF (MS850) was purchased from NeoMarkers (Lab Vision, Fremont, CA). Antibodies for CDK4 (DCS156), CDK6 (DCS83), cyclin D1 (DSS6), cyclin D3 (DCS22) and p27KIP1 (2552) were purchased from Cell Signaling Technology (Danvers, MA). Anti-GAPDH was purchased from Applied Biosystems. Anti-FLAG (A9469) and Anti-HA (H9658) were purchased from Sigma. A Western blue stabilized substrate was used to detect the signals originated from alkaline phosphatase in accordance with the manufacturer's protocol (Promega).

WST-1 assay. After two successive siRNA transfection (days 0 and 1), cells were inoculated into a 24 -well plate at a concentration of $1-2 \times 10^{4}$ cells/well (day 2). A WST-1 assay was then performed (days 3, 4 and 6), according to the manufacturer's protocol. For the detection of FBXL16 effects on cell proliferation, cells were transfected with expression vectors (day 0 ), and cells were inoculated into a 24-well plate at a concentration of $5 \times 10^{2}$ cells/well (day 1 ). A WST-1 assay was then performed (days 2-6), according to the manufacturer's protocol. Alternatively, cells were inoculated into a 24-well plate at a concentration of $5 \times 10^{2}$ cells/well on day 2 of siRNA transfection protocol. A WST-1 assay was then performed (days 4-7), according to the manufacturer's protocol. Briefly, a WST-1 solution was added to each well, followed by $1 \mathrm{~h}$ of incubation at $37^{\circ} \mathrm{C}$, and the absorbance was measured at $450 \mathrm{~nm}$. The reference wavelength was $600 \mathrm{~nm}$. Cell viability was calculated as the absorbance in the treated cells over the control. Statistical differences were analyzed using a two-tailed Student's t-test. A value of $\mathrm{P}<0.05$ ( $n=3$ or 4 ) was considered to indicate a statistically significant difference.

Caspase-3/7 assay. The induction of apoptosis was assessed using a Caspase-Glo 3/7 assay according to the manufacturer's instructions (Promega). In brief, on day 2 of siRNA transfection protocol, the cells were inoculated into a 96-well plate at a concentration of $1 \times 10^{4}$ cells/well $24 \mathrm{~h}$ before treatment. The cells were then treated with chemicals (on day 3 ) for $48 \mathrm{~h}$, followed by $1 \mathrm{~h}$ of incubation with Caspase-Glo 3/7 substrate at room temperature, and the resulting activity was measured using a GloMax 20/20n Luminometer (Promega). The activity was presented as relative light units (RLU, x106) in the treated cells relative to the control value (treated with $0.1 \%$ DMSO). Blanks were measured in wells containing $0.1 \%$ DMSO or chemicals without cells.

Luciferase assay. For the promoter assay, $2 \times 10^{4}$ cells were transfected with FuGENE6 (Roche, Basel, Switzerland), in accordance with the manufacturer's instructions. Briefly, $200 \mathrm{ng}$ of the expression plasmid (pcDNA3 or pcDNA3E2F1), $200 \mathrm{ng}$ of the firefly luciferase reporter plasmids pGL3-Basic (Promega) and 0.6 ng of the Renilla luciferase reporter plasmid pRL-TK (Promega) per 24-well dish were used for each transfection. Cells were lysed $24 \mathrm{~h}$ after transfection by applying $100 \mu \mathrm{l}$ Passive Lysis Buffer of the Dual Luciferase Reporter Assay Kit (Promega) into each well of the 24-well plate. Cell lysate $(5 \mu \mathrm{l})$ was used for the luciferase reporter assay with the same kit according to the manufacturer's protocol. Light intensity was quantified in a GloMax 20/20n Luminometer (Promega). Experiments were performed at least in triplicate. As control for the transfection efficiency, the firefly luciferase activity values were normalized to the Renilla luciferase activity values. Data are presented as mean values \pm S.D. Statistical differences were analyzed using two-tailed Student's t-tests. A value of $\mathrm{P}<0.05$ $(n=3)$ was considered to indicate a statistically significant difference.

Oligonucleotide microarray analysis. On day 2 of siRNA transfection protocol, total RNA samples were prepared using RNeasy mini-spin column (Qiagen, Valencia, CA). The quality of these RNA samples was examined using Agilent 2100 Bioanalyzer (Agilent Technologies, Palo Alto, CA) to ensure the integrity of RNA samples before use. Fluorescent labeled cRNA targets were generated from $500 \mathrm{ng}$ of total RNA following the protocol in the user's manual (Agilent Technologies). Both Cyanine 3-labeled CTP and 5-labeled CTP (10 mM) were purchased from Perkin-Elmer/NEN Life Science (Boston, MA). RNA amplification and labeling was performed using the Low RNA Fluorescent Linear Amplification Kit Plus (Agilent Technologies). Briefly, T7 promoter primer $(1.2 \mu \mathrm{l})$ was added to total RNA. Final volume is brought to $9.5 \mu \mathrm{l}$ with nuclease-free water. Each $2 \mu \mathrm{l}$ of Spike A and Spike B was added to tubes, which will be labeled by $\mathrm{Cy} 3$ and $\mathrm{Cy} 5$, respectively. The primer and the RNA template were denatured at $65^{\circ} \mathrm{C}$ in a heating block for $10 \mathrm{~min}$ and cooled on ice for $5 \mathrm{~min}$. A volume of $8.5 \mu \mathrm{l}$ of cDNA mix was added to each reaction tube. The cDNA 
synthesis reaction mix contains $4 \mu 1$ of $5 X$ First Strand Buffer, $2 \mu \mathrm{l}$ of $0.1 \mathrm{M}$ DTT, $1 \mu \mathrm{l}$ of $10 \mathrm{mM}$ dNTP mix, $1 \mu \mathrm{l}$ of MMLV reverse transcriptase, and $0.5 \mu 1$ of RNaseOUT. Samples were incubated at $40^{\circ} \mathrm{C}$ in a circulating water bath for $2 \mathrm{~h}$, heated at $65^{\circ} \mathrm{C}$ for $15 \mathrm{~min}$, and cooled on ice for $5 \mathrm{~min}$. A volume of $2.4 \mu \mathrm{l}$ of $10 \mathrm{mM}$ cyanine 3-CTP (for control sample) or cyanine 5-CTP (for p16INK4A- or p14ARFsilenced sample) was added to each sample tube, following by the addition of $57.6 \mu 1$ of transcription master mix. The transcription master mix contains $15.3 \mu \mathrm{l}$ of nuclease-free water, $20 \mu 1$ of $4 \mathrm{X}$ transcription buffer, $6 \mu \mathrm{l}$ of $0.1 \mathrm{M}$ DTT, $8 \mu \mathrm{l}$ of NTP mix, $6.4 \mu \mathrm{l}$ of $50 \%$ PEG, $0.5 \mu 1$ of RNaseOUT, $0.6 \mu 1$ of inorganic pyrophosphatase, and $0.8 \mu 1$ of T7 RNA polymerase. The reaction mix was then incubated at $40^{\circ} \mathrm{C}$ in the dark for $2 \mathrm{~h}$. Labeled cRNA was purified using RNeasy mini-spin columns (Qiagen). The cRNA product was eluted twice with nuclease-free water from the column in $60 \mu \mathrm{l}$ of final volume. Yields of cRNA were determined by UV spectrophotometry.

Hybridization was performed using Gene Expression Hybridization Kit (Agilent Technologies) following Agilent's user's manual. The labeled cRNA $(0.75 \mu \mathrm{g})$ per channel was mixed with $50 \mu \mathrm{l}$ of $10 \mathrm{x}$ blocking agent and nuclease-free water to a final volume of $240 \mu \mathrm{l}$. Then $10 \mu \mathrm{l}$ of $25 \mathrm{X}$ fragmentation buffer was added to each sample tube and the reactions were incubated at $60^{\circ} \mathrm{C}$ in the dark for $30 \mathrm{~min}$. The addition of $250 \mu \mathrm{l}$ of $2 \mathrm{X}$ hybridization buffer terminated the reaction. A $500 \mu 1$ volume of the hybridization mix was applied to each of 60 mer Human1A v2 Oligonucleotide Microarray (G4110B, Agilent Technologies) and hybridized in a hybridization oven at $65^{\circ} \mathrm{C}$ for $17 \mathrm{~h}$. The slides were disassembled in Gene Expression Wash Pack (Agilent Technologies). They were washed with Wash buffer 1 for $1 \mathrm{~min}$ at room temperature and Wash buffer 2 for $1 \mathrm{~min}$ at $37^{\circ} \mathrm{C}$, then with acetonitrile for $1 \mathrm{~min}$, and air dried.

The arrays were scanned by the Agilent dual-laser DNA microarray scanner with $100 \mu \mathrm{m}$ resolution using SureScan technology, extracted by Feature Extraction software, and analyzed by Rosetta Resolver software. An average of three replicate samples was used for each experiment.

\section{Results}

SiRNA-mediated knockdown of p16INK4A and p14ARF. To check the specificity of the siRNA for p16INK4A (siRNAs 240 and 319) and for p14ARF (siRNAs 169 and 279) (Fig. 1A), first of all, we searched for suitable cells in which detectable amounts of p16INK4A and p14ARF are expressed. Using a specific antibody for p16INK4A and p14ARF, we performed a Western blot analysis. Among the cultured cells that were examined, a cell lysate prepared from HeLa cells had detectable protein levels of p16INK4A and p14ARF, whereas those from A549 or WI-38 cells failed to show any detectable protein levels for p16INK4A and p14ARF (Fig. 1B). Functional studies on both p16INK4A and p14ARF proteins in HeLa cells have been well-documented elsewhere (13-16), indicating that HeLa cells possess functional p16INK4A and p14ARF proteins. Therefore, we decided to use HeLa cells in our further studies. SiRNAs for p16INK4A (siRNAs 240 and 319) and for p14ARF (siRNAs 169 and 279) were introduced into HeLa cells on two successive days (days 0 and 1); on day 3 , the cell lysates were checked using individual antibodies. Compared with the control siRNA-transfected cells, p16INK4A siRNAs (240 and 319) suppressed the p16INK4A protein but not the p14ARF protein, while p14ARF siRNAs (169 and 279) efficiently silenced the p14ARF protein but not the p16INK4A protein, indicating that these siRNAs are specific for each of the mRNAs (Fig. 1C).

In the cervical cell lines $\mathrm{SiHa}$ and Cas $\mathrm{Ki}, \mathrm{p} 16 \mathrm{INK} 4 \mathrm{~A}$ silencing resulted in increased protein levels of cell cycle regulators such as pRb, p53 and p21CIP1/WAF1, even at $12 \mathrm{~h}$ after siRNA transfection and up to $48 \mathrm{~h}$ after siRNA transfection (16). To determine whether p16INK4A and p14ARF inactivation resulted in the upregulation of these cell cycle regulators in HeLa cells, we performed a Western blot analysis using cell extracts obtained on day 2 of the siRNA transfection protocol. None of the proteins that were examined, including p53, p53 Ser15, p21WAF1/CIP1, MDM2, CDK4, CDK6, cyclin D1, cyclin D3 and GAPDH (glyceraldehyde-3-phosphate dehydrogenase), were upregulated in the p16INK4A- and p14ARF-silenced HeLa cells (Fig. 1D). In mouse embryonic fibroblasts deficient for p16Ink4a, the p15Ink4b protein level is elevated in a compensatory manner under conditions of stress (17). To reproduce the long-term effect of p16INK4A loss, we checked the effect of p16INK4A and p14ARF silencing in HeLa cells until day 7 of the siRNA transfection protocol. Both p16INK4A and p14ARF siRNA suppressed the p16INK4A and p14ARF proteins, respectively, up until day 7 , compared with the levels in the control siRNA-treated cells (Fig. 1E). On day 6 of the siRNA transfection protocol, the p15INK4B protein level was slightly increased in the p16INK4A-silenced cells but not in the p14ARF-silenced cells (Fig. 1F).

To see whether these upstream regulators can affect the INK4A/ARF locus in HeLa cells, we overexpressed Bmi-1 (pF-Bmi), CDC6 (pcGN-HA-CDC6) and oncogenic HRAS (pRK5-V12 HRAS), and checked the protein levels for p16INK4A, but no significant changes were observed (Fig. 1G).

Rapid reduction of p16INK4A and p14ARF proteins had no effect on cellular phenotype. To maximize the effect of p16INK4 and p14ARF inactivation, we transfected both p16INK4 and p14ARF siRNA simultaneously. The downregulation of both p16INK4 and p14ARF proteins was confirmed by a Western blot analysis (Fig. 2A); however, cell growth/ proliferation based on a WST-1 assay showed no changes compared with control siRNA-treated cells on days 3, 4 and 6 of the siRNA transfection protocol (Fig. 2B). In SiHa cells, p16INK4A silencing resulted in increased populations of apoptotic cells (16). To determine whether etoposide-induced apoptosis is affected by p16INK4A silencing in HeLa cells, we assessed caspase-3/7 activity. Etoposide-induced apoptosis in HeLa cells showed no apparent differences in caspase-3/7 activity among p16INK4A, p14ARF and control siRNA-treated cells, whereas an etoposide concentrationdependent upregulation of caspase-3/7 activity was manifested (Fig. 3A). In addition, etoposide efficiently induced p53 protein accumulation accompanied by an elevation in Ser15 phosphorylation (Fig. 3B). 


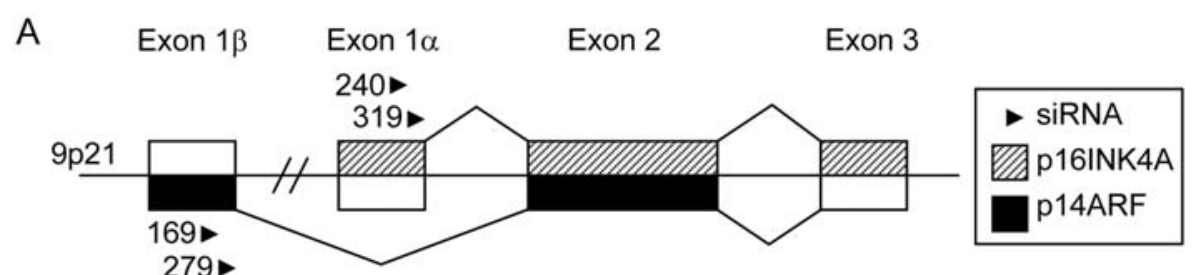

B

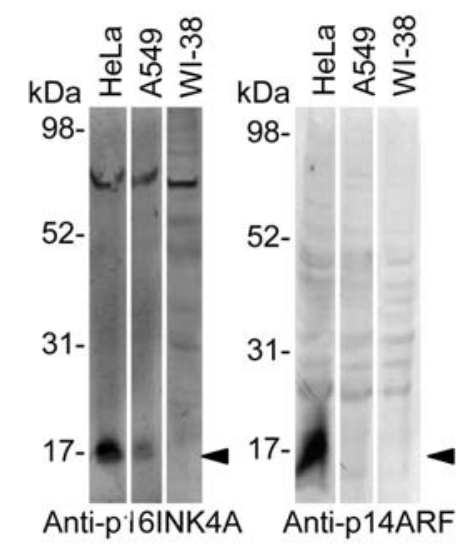

D
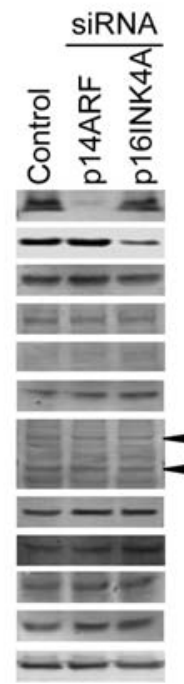

Anti-p14ARF

Anti-p16INK4A

Anti-p15INK4B

Anti-p53

Anti-p53 Ser15

Anti-p21WAF1/CIP1

- Anti-MDM2 (p90)

Anti-MDM2 (cleaved p60)

Anti-CDK4

Anti-CDK6

Anti-Cyclin D1

Anti-Cyclin D3

Anti-GAPDH

F

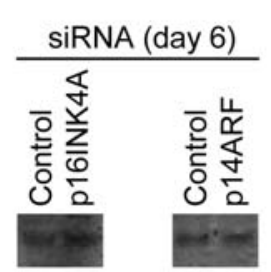

Anti-p15INK4B
C

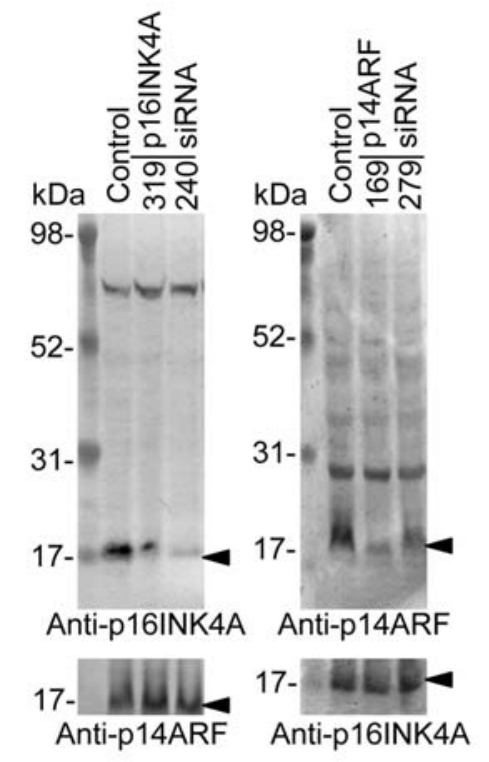

E

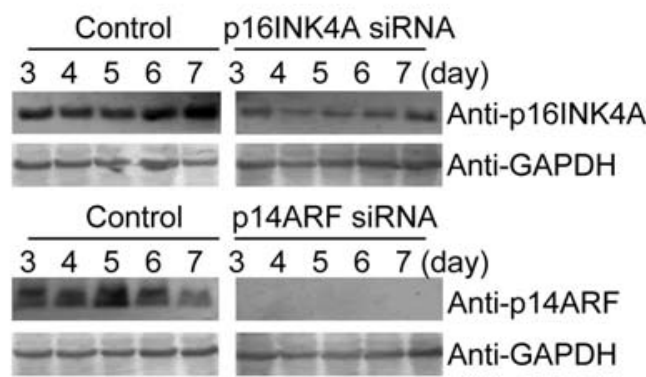

Figure 1. Silencing of p16INK4A and p14ARF in human cultured cells. (A) Schematic representation of INK4a/ARF locus in chromosome 9p21. The exons $(1 \beta, 1 \alpha, 2$ and 3$)$ are indicated by the boxes. The exons used for p16INK4A and p14ARF are indicated by the gray and black boxes, respectively. The arrowhead indicates the position of siRNA. (B) Western blot analysis of p16INK4A and p14ARF. Cell lysates $(100 \mu \mathrm{g})$ were extracted from the indicated cells and blotted with anti-p16INK4A (left panel) and anti-p14ARF (right panel). Molecular weight (kDa) is indicated on the left side of each panel. The arrowheads indicate the positions of p16INK4A (left panel) and p14ARF (right panel). (C) Silencing of p16INK4A (left panel) and p14ARF (right panel) in HeLa cells. On day 2 of the siRNA transfection protocol, the cell lysates were extracted and blotted with anti-p16INK4A and anti-p14ARF. Two different siRNAs were used for p16INK4A (240 and 319) and p14ARF (169 and 279). The molecular weight (kDa) is indicated on the left side of each panel. The arrowheads indicate the positions of p16INK4A (left upper and right lower panels) and p14ARF (right upper and left lower panels). (D) Protein levels of the cell cycle regulators in p16INK4A- and p14ARF-silenced HeLa cells. On day 2 of the siRNA transfection protocol, the cell lysates were extracted and blotted with the indicated antibodies. (E) Time-course analysis of the silencing effect of p16INK4A and p14ARF siRNA in HeLa cells. On days 3 to 7 of the siRNA transfection protocol, the cell lysates were extracted and blotted with the indicated antibodies. (F) Expression level of p15INK4B in p16INK4A- and p14ARFsilenced HeLa cells. On day 6 of the siRNA transfection protocol, the cell lysates were extracted and blotted with anti-p15INK4B. (G) Effect of upstream regulators on p16INK4A protein level. Expression vectors for Bmi-1 (Left panel), CDC6 (middle panel) and oncogenic HRAS (right panel) were transfected into HeLa cells; after $72 \mathrm{~h}$, the cell lysates were extracted and blotted with the indicated antibodies. 
A

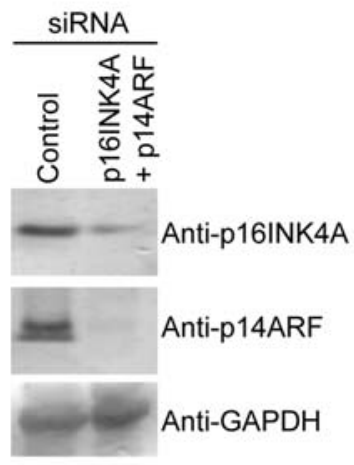

B

Control siRNA

p16INK4A + p14ARF siRNA

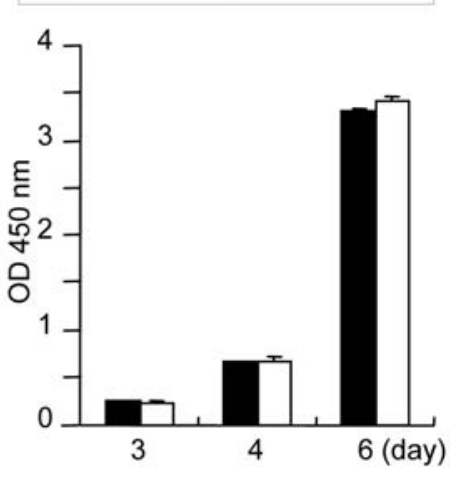

Figure 2. Effect of p16INK4A and p14ARF double silencing on cell proliferation rate in HeLa cells. (A) Western blot analysis of p16INK4A and p14ARF in cells transfected with both p16INK4A and p14ARF siRNAs. On day 2 of the siRNA transfection protocol, the cell lysates were extracted and blotted with the indicated antibody. (B) WST-1 assay of p16INK4A and p14ARF double-silenced HeLa cells on days 3, 4 and 6 of the siRNA transfection protocol. The black and white bars indicate the OD 450-nm value of the control and p16INK4A plus p14ARF siRNA-treated cells, respectively. Values are expressed as the means \pm S.D. $(n=4)$.
DNA microarray analysis of p16INK4A- and p14ARFsilenced HeLa cells. We next examined whether any changes in gene expression occurred in p16INK4A knockdown or p14ARF knockdown cells, compared with in control siRNAtreated cells. After siRNA transfection for two successive days (days 0 and 1), the total RNA was extracted and used in a DNA microarray analysis (day 2). Surprisingly, the expression levels of several genes were altered in a similar manner in the p16INK4A-knockdown cells and the p14ARF-knockdown cells (Tables I-IV). As commonly regulated genes in the p16INK4A- and p14ARF-knockdown cells, FBXL16 (F-box and leucine-rich repeat protein 16), EIF4EBP2 (eukaryotic translation initiation factor $4 \mathrm{E}$ binding protein 2), SIM2 (single-minded homolog 2, Drosophila), ARMCX6 (armadillo repeat containing, X-linked 6), and POLR2L (polymerase (RNA) II (DNA directed) polypeptide L, $7.6 \mathrm{kDa}$ ) mRNAs were upregulated (Tables I and III), while CCL2 [chemokine (C-C motif) ligand 2], CYP4F11 (cytochrome P450, family 4, subfamily F, polypeptide 11), and CYP4F2 (cytochrome P450, family 4, subfamily F, polypeptide 2) mRNAs were downregulated (Tables II and IV). We focused on the novel genes that were upregulated in both p16INK4A- and p14ARFknockdown cells. To further examine the reproducibility of the DNA microarray results, we performed RT-PCR analysis of FBXL16, EIF4EBP2 and ARMCX6 genes. These genes were increasingly transcribed shortly after p16INK4 and p14ARF inactivation in HeLa cells, whereas GAPDH was equally detected in p16INK4-specific siRNA-, p14ARFspecific siRNA-, and control siRNA-treated cells (Fig. 4A). Interestingly, the expression pattern for FBXL16 in various human adult tissues revealed that FBXL16 mRNA is expressed in a tissue-dependent manner in areas such as the brain, liver, pancreas, spleen and testis, compared with the results obtained for EIF4EBP2 (Fig. 4B). No FBXL16 transcript was detected in fetal tissues in an RT-PCR assay, even after 40 cycles of amplification (Fig. 4B).

Transcriptional regulation of FBXL16. Apparently, p16INK4 and p14ARF do not directly affect the gene expression levels $\mathrm{B}$
A

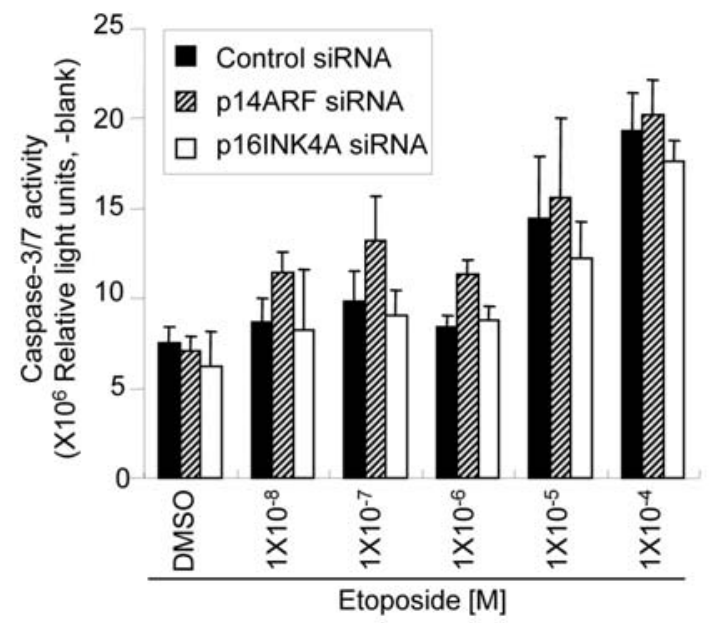

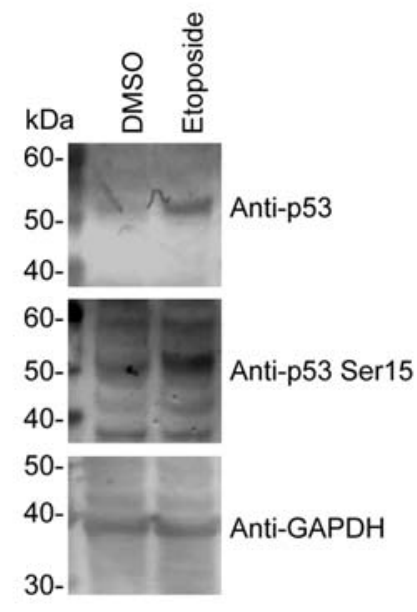

Figure 3. Effect of p16INK4A and p14ARF silencing on etoposide-induced apoptosis in HeLa cells. (A) On day 3 of the siRNA transfection protocol, the cells were exposed to the indicated concentrations of etoposide for $48 \mathrm{~h}$ and the caspase-3/7 activity was measured. The black, gray and white bars indicate the caspase-3/7 activity (relative right units) in the control, p14ARF and p16INK4A siRNA-treated cells, respectively. Values are expressed as the means \pm S.D. (n=6). (B) Western blot analysis of p53 protein and its Ser15 phosphorylation level after etoposide treatment. Etoposide (1x10-5 M) was added to the cell culture for $48 \mathrm{~h}$. The molecular weight $(\mathrm{kDa})$ is shown on the left side of each panel. 
Table I. Messenger RNAs upregulated in p16INK4A-knocked down HeLa cells.

\begin{tabular}{llll}
\hline Gene symbol & GenBank & Fold change & \\
\hline FBXL16 $^{a}$ & NM_153350 & 3.4 & Fene name \\
EIF4EBP2 $^{\text {a }}$ & NM_004096 & 3.3 & Eukaryotic translation initiation factor 4E binding protein 2 \\
SIM2 $^{\mathrm{a}}$ & NM_005069 & 2.4 & Single-minded homolog 2 \\
MSI2 & NM_170721 & 2.3 & Musashi homolog 2 \\
ARMCX6 $^{\mathrm{a}}$ & NM_019007 & 2.3 & Armadillo repeat containing, X-linked 6 \\
POLR2L & NM_021128 & 2.2 & Polymerase (RNA) II (DNA directed) polypeptide L \\
KRT86 & NM_002284 & 2.2 & Keratin 86 \\
M-RIP & NM_015134 & 2.1 & Myosin phosphatase-Rho interacting protein \\
STARD4 & NM_139164 & 2.0 & START domain containing 4, sterol regulated \\
TM4SF20 & NM_024795 & 2.0 & Transmembrane 4 L six family member 20 \\
\hline
\end{tabular}

${ }^{a}$ Commonly upregulated genes also in p14ARF-knocked down HeLa cells.

Table II. Messenger RNAs downregulated in p16INK4A-knocked down HeLa cells.

\begin{tabular}{|c|c|c|c|}
\hline Gene symbol & GenBank & Fold change & Gene name \\
\hline ASNS & NM_001673 & 3.5 & Asparagine synthetase \\
\hline ALDH1L1 & NM_012190 & 3.1 & Aldehyde dehydrogenase 1 family, member L1 \\
\hline HIST1H2BK & NM_080593 & 2.8 & Histone cluster $1, \mathrm{H} 2 \mathrm{bk}$ \\
\hline CCL2 $2^{\mathrm{a}}$ & NM_002982 & 2.7 & Chemokine (C-C motif) ligand 2 \\
\hline STARD3 & NM_006804 & 2.7 & START domain containing 3 \\
\hline SCARA3 & NM_016240 & 2.5 & Scavenger receptor class A, member 3 \\
\hline P2RY6 & NM_176798 & 2.5 & Pyrimidinergic receptor P2Y, G-protein coupled, 6 \\
\hline IFI27 & NM_005532 & 2.3 & Interferon, $\alpha$-inducible protein 27 \\
\hline SCARB2 & NM_005506 & 2.3 & Scavenger receptor class $\mathrm{B}$, member 2 \\
\hline CYP4F11 & NM_021187 & 2.3 & Cytochrome P450, family 4, subfamily F, polypeptide 11 \\
\hline CTGF & NM_001901 & 2.3 & Connective tissue growth factor \\
\hline MAN2A1 & NM_002372 & 2.3 & Mannosidase, $\alpha$, class $2 \mathrm{~A}$, member 1 \\
\hline CYP4F2 $2^{\text {a }}$ & NM_001082 & 2.2 & Cytochrome P450, family 4, subfamily F, polypeptide 2 \\
\hline SNX17 & NM_014748 & 2.2 & Sorting nexin 17 \\
\hline CD99 & NM_002414 & 2.2 & CD99 molecule \\
\hline PPL & NM_002705 & 2.2 & Periplakin \\
\hline ZDHHC20 & NM_153251 & 2.1 & Zinc finger, DHHC-type containing 20 \\
\hline LOC202459 & NM_145303 & 2.1 & LOC202459 \\
\hline RRM2B & NM_015713 & 2.0 & Ribonucleotide reductase M2 B \\
\hline CDH16 & NM_004062 & 2.0 & Cadherin 16, KSP-cadherin \\
\hline MKRN1 & NM_013446 & 2.0 & Makorin, ring finger protein, 1 \\
\hline ACVR2B & NM_001106 & 2.0 & Activin A receptor, type IIB \\
\hline DPM1 & NM_003859 & 2.0 & Dolichyl-phosphate mannosyltransferase polypeptide 1 , catalytic subunit \\
\hline TAGLN2 & NM_003564 & 2.0 & Transgelin 2 \\
\hline PPP5C & NM_006247 & 2.0 & Protein phosphatase 5 , catalytic subunit \\
\hline
\end{tabular}

aCommonly downregulated genes also in p14ARF-knocked down HeLa cells.

of downstream genes. In the current model, p16INK4 and p14ARF are upstream regulators of E2F1 and p53, respectively. To further investigate how these transcriptional factors regulate the genes that are upregulated in both p16INK4A- and p14ARF-knockdown cells, we examined the changes in mRNA expression in E2F1-overexpressing HeLa cells using RT-PCR analysis. For this purpose, we focused on the FBXL16 gene. We transfected an E2F1 expression plasmid 
Table III. Messenger RNAs upregulated in p14ARF-knocked down HeLa cells.

\begin{tabular}{|c|c|c|c|}
\hline Gene symbol & GenBank & Fold change & Gene name \\
\hline FBXL16 ${ }^{\mathrm{a}}$ & NM_153350 & 2.9 & F-box and leucine-rich repeat protein 16 \\
\hline KIAA0408 & NM_014702 & 2.7 & KIAA0408 \\
\hline SIM2a & NM_005069 & 2.6 & Single-minded homolog 2 \\
\hline EIF4EBP2 ${ }^{\mathrm{a}}$ & NM_004096 & 2.6 & Eukaryotic translation initiation factor 4E binding protein 2 \\
\hline KIAA1446 & NM_020836 & 2.5 & Brain-enriched guanylate kinase-associated protein \\
\hline TXNIP & NM_006472 & 2.5 & Thioredoxin interacting protein \\
\hline NCOR2 & NM_006312 & 2.5 & Nuclear receptor co-repressor 2 \\
\hline ZNF219 & NM_016423 & 2.5 & Zinc finger protein 219 \\
\hline GLIPR1 & NM_006851 & 2.4 & GLI pathogenesis-related 1 \\
\hline POLR2L ${ }^{a}$ & NM_021128 & 2.3 & Polymerase (RNA) II (DNA directed) polypeptide L \\
\hline MARVELD1 & NM_031484 & 2.2 & MARVEL domain containing 1 \\
\hline FOXF2 & NM_001452 & 2.1 & Forkhead box F2 \\
\hline VDR & NM_001017535 & 2.1 & Vitamin D (1,25- dihydroxyvitamin D3) receptor \\
\hline RUSC1 & NM_014328 & 2.1 & RUN and SH3 domain containing 1 \\
\hline CCDC80 & NM_199511 & 2.1 & Coiled-coil domain containing 80 \\
\hline ARMCX6 $^{\mathrm{a}}$ & NM_019007 & 2.1 & Armadillo repeat containing, X-linked 6 \\
\hline MLXIP & NM_014938 & 2.1 & MLX interacting protein \\
\hline TRIM14 & NM_014788 & 2.0 & Tripartite motif-containing 14 \\
\hline
\end{tabular}

${ }^{a}$ Commonly upregulated genes also in p16INK4A-knocked down HeLa cells.

Table IV. Messenger RNAs downregulated in p14ARF-knocked down HeLa cells.

\begin{tabular}{|c|c|c|c|}
\hline Gene symbol & GenBank & Fold change & Gene name \\
\hline MX1 & NM_002462 & 5.5 & Myxovirus (influenza virus) resistance 1 \\
\hline CCDC56 & NM_001040431 & 3.1 & Coiled-coil domain containing 56 \\
\hline CYP4F11 ${ }^{\mathrm{a}}$ & NM_021187 & 2.7 & Cytochrome P450, family 4, subfamily F, polypeptide 11 \\
\hline C16orf75 & NM_152308 & 2.6 & Chromosome 16 open reading frame 75 \\
\hline CPEB1 & NM_030594 & 2.5 & Cytoplasmic polyadenylation element-binding protein 1 \\
\hline LYN & NM_002350 & 2.5 & v-yes-1 Yamaguchi sarcoma viral-related oncogene homolog \\
\hline FUT1 & NM_000148 & 2.4 & Fucosyltransferase 1 (galactoside $2-\alpha$-L-fucosyltransferase, H blood group) \\
\hline CKS1B & NM_001826 & 2.3 & CDC28 protein kinase regulatory subunit 1B \\
\hline CYP4F2a & NM_001082 & 2.3 & Cytochrome P450, family 4, subfamily F, polypeptide 2 \\
\hline UBE2G1 & NM_003342 & 2.3 & Ubiquitin-conjugating enzyme E2G 1 \\
\hline ATP8B3 & NM_138813 & 2.3 & ATPase, class I, type 8B, member 3 \\
\hline PGLS & NM_012088 & 2.3 & 6-phosphogluconolactonase \\
\hline FGFR3 & NM_000142 & 2.2 & Fibroblast growth factor receptor 3 \\
\hline ATP2A2 & NM_001681 & 2.2 & ATPase, $\mathrm{Ca}^{++}$transporting, cardiac muscle, slow twitch 2 \\
\hline LRP8 & NM_033300 & 2.2 & Low density lipoprotein receptor-related protein 8 , apolipoprotein e receptor \\
\hline TM4SF1 & NM_014220 & 2.2 & Transmembrane 4 L six family member 1 \\
\hline CHMP6 & NM_024591 & 2.1 & Chromatin-modifying protein 6 \\
\hline AADACL1 & NM_020792 & 2.1 & Arylacetamide deacetylase-like 1 \\
\hline CCL2 $^{\mathrm{a}}$ & NM_002982 & 2.1 & Chemokine (C-C motif) ligand 2 \\
\hline SLC25A1 & NM_005984 & 2.1 & Solute carrier family 25 (mitochondrial carrier; citrate transporter), member 1 \\
\hline ALDH3A1 & NM_000691 & 2.1 & Aldehyde dehydrogenase 3 family, memberA1 \\
\hline CYP4F8 & NM_007253 & 2.1 & Cytochrome P450, family 4, subfamily F, polypeptide 8 \\
\hline PYGB & NM_002862 & 2.1 & Phosphorylase, glycogen; brain \\
\hline
\end{tabular}

${ }^{\mathrm{a} C o m m o n l y ~ d o w n r e g u l a t e d ~ g e n e s ~ a l s o ~ i n ~ p 16 I N K 4 A-k n o c k e d ~ d o w n ~ H e L a ~ c e l l s . ~}$ 
A

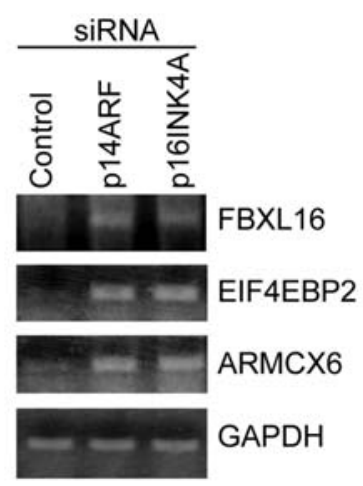

B

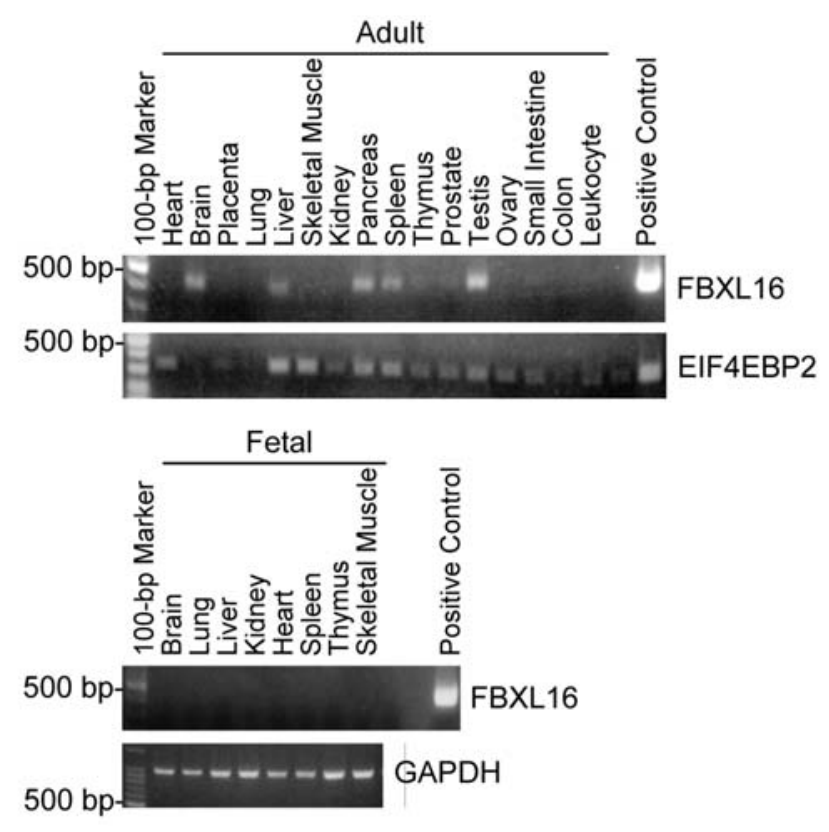

Figure 4. RT-PCR analysis of candidate genes regulated by p16INK4A and p14ARF. (A) On day 2 of the siRNA transfection protocol, the total RNA was extracted and detected using the indicated gene primer set and RT-PCR. The numbers of PCR cycles for EBXL16, EIF4EBP2, ARMCX6 and GAPDH were 40, 22, 22 and 18, respectively. (B) Tissue distribution of FBXL16 mRNA. cDNA derived from the indicated human adult (upper panel) and fetal (lower panel) tissues were used as templates for PCR. The expression levels of EIF4EBP2 and GAPDH are also shown. The 100-bp marker and the highlighted 500-bp band are shown on the left side of each panel. The positive controls for EBXL16 and EIF4EBP2 were EST (0.1 ng of 5262152) and HeLa cDNA, respectively. The numbers of PCR cycles for EBXL16, EIF4EBP2 and GAPDH were 40, 28 and 25, respectively.

into HeLa cells (Fig. 5A), and the total RNA was examined using RT-PCR. E2F1 clearly upregulated the FBXL16 expression level, compared with that in mock-transfected cells, whereas the GAPDH level was unchanged in the mockand E2F1-transfected cells (Fig. 5B). The overexpression of p53 failed to induce the expression of FBXL16 (data not shown).

Next, we attempted to analyze the promoter region of the FBXL16 gene. For this purpose, we amplified the putative promoter region of FBXL16 encompassing the -646 to +44 region $(-646 /+44)$, in which the transcriptional start site was designated as +1 , using PCR and then cloned it into a pGL3Basic luciferase reporter plasmid (Fig. 5C). The amplified region contained multiple consensus sites for E2F binding, as predicted using Transfac software when the threshold was set to 70 (Fig. 5C). The pGL3 $-646 /+44$ reporter showed an approximately 10 -fold induction of luciferase activity, compared with that in pGL3-Basic or pGL3 -646/+44 cotransfected with pcDNA3 (Fig. 5D). Other activator E2Fs could induce luciferase activity, whereas suppressor E2Fs failed to induce the luciferase activity of pGL3 -646/+44 (Fig. 5D). Transfac predicted that two highly-matched consensus sequences were located at the $5^{\prime}$ and $3^{\prime}$ ends of the $-646 /+44$ region. To assess which site was responsible for luciferase induction by E2F1, we constructed reporter plasmids with deletions at the $5^{\prime}$ and 3 ' ends: pGL3 $-579 /+44$, $-646 /-90$ and $-579 /-90$ (Fig. 5C). The luciferase activities of these reporter plasmids were still upregulated by E2F1 cotransfection (Fig. 5E), indicating that other E2F consensus sites recognized in the $-579 /-90$ region might have been bound by E2F1. Therefore, we constructed reporter plasmids with the 5 ' or 3 ' half part of pGL3 -579/-90, pGL3 -579/-287 and $-306 /-90$, respectively (Fig. 5C). The luciferase activities of these reporter plasmids were again upregulated by E2F1 co-transfection (Fig. 5E). Among the reporter plasmids, pGL3 -579/-287 solely lacked the basal promoter activity identical to that obtained by pGL3-Basic (Fig. 5F), suggesting FBXL16 gene responsiveness to E2F1 is independent of basal promoter activity.

Functional analysis of FBXL16. Based on the amino acid composition of FBXL16, this protein is thought to function as a component of SCF ubiquitin ligase (18). Therefore, we checked to see if FBXL16 overexpression had any effect on cell proliferation. To this end, we constructed an N-terminally FLAG-tagged FBXL16 expression vector and overexpressed it in HeLa cells. The anti-FLAG antibody clearly detected a predominant band of FLAG-tagged FBXL16 protein after $48 \mathrm{~h}$ of transfection (Fig. 6A, left panel). FBXL16 stable transformants could not be recovered during G418 selection, whereas pcDNA3 control transformants survived, suggesting that FBXL16 might have toxic effects on cell proliferation. Therefore, we monitored cell proliferation using a WST-1 assay; however, no apparent difference was observed on days $0-6$, where day 6 was the last day on which cell proliferation was measured (data not shown).

Next we intended to reduce the FBXL16 protein by RNAi. For this, we introduced FBXL16-specific siRNA (days 0 and 1) and FLAG-tagged FBXL16 expression vector (day 2) into the cells. We checked the FBXL16 protein level on day 4 and monitored cell proliferation rate on day 4 - day 7 by WST-1 assay. The FLAG-tagged FBXL16 protein was 
A

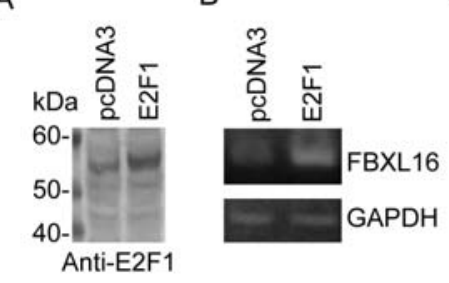

D

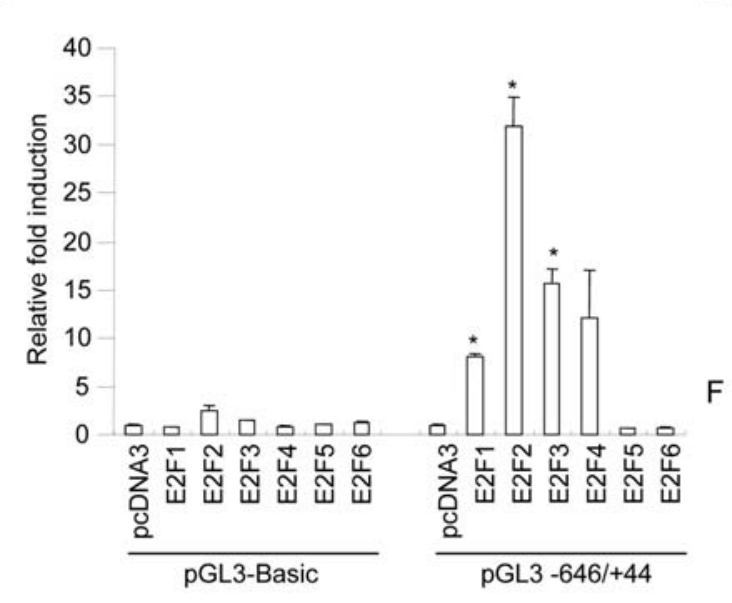

$\mathrm{F}$
C

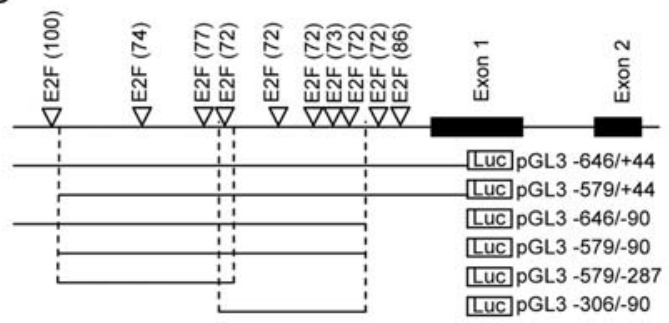

$\mathrm{E}$

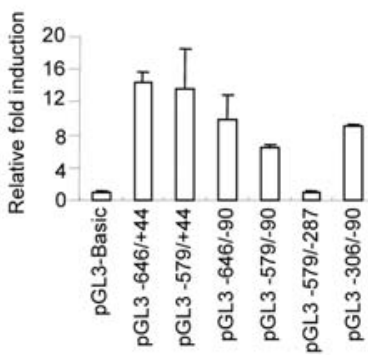

Figure 5. Effect of E2F1 overexpression on FBXL16 transcription. (A) HeLa cells were transfected with pcDNA3 or pcDNA3-E2F1. After 48 h, the cell lysates were extracted and blotted with anti-E2F1. The molecular weight (kDa) is shown on the left side of each panel. (B) RT-PCR of $F B X L 16$ mRNA in E2F1-transfected cells. GAPDH mRNA was also detected as a control. The number of PCR cycles for EBXL16 was 40. (C) Promoter region of FBXL16 gene. The black boxes indicate exons 1 and 2 of the FBXL16 gene. The inverted triangles indicate the relative position of the E2F consensus elements predicted by the Transfac program with the calculated threshold shown in parentheses. Luc indicates the luciferase gene. (D) Luciferase reporter activity induced by E2Fs. Relative-fold induction was shown as the value obtained when pcDNA3 co-transfection was defined as 1 . Values are expressed as the means \pm S.D. ( $\mathrm{n}=3$ ). ${ }^{*} \mathrm{P}<0.05$ (compared with pcDNA3-transfected cells), using a two-tailed Student $\mathrm{t}$-test. (E) Luciferase reporter activity of various promoter region of FBXL16 gene induced by E2F1. The value of E2F1 co-transfection was divided by the value of pcDNA3 co-transfection, then the obtained value was shown as relative-fold induction when pGL3 $-646 /+44$ was defined as 100 . Values are expressed as the means \pm S.D. (n=3). (F) Luciferase reporter activity of various promoter region of FBXL16 gene. Relative-fold induction was shown as the value of pGL3-Basic defined as 1. Values are expressed as the means \pm S.D. $(\mathrm{n}=3)$.

reduced to half level by FBXL16-specific siRNA when compared to control siRNA, whereas GAPDH was unaffected by FBXL16-specific siRNA (Fig. 6A, middle and right panels). The WST-1 assay showed that FBXL16 knockdown increased the cell proliferation rate of HeLa cells (Fig. 6B). It is well-known that F-box proteins are involved in the timely degradation process of cell cycle regulators. For example, FBXL1 (alias SKP2) has a role in ubiquitin-mediated degradation of p27KIP1 (19). We checked whether FBXL16 knockdown affects the protein level of cell cycle regulators including p27KIP1, CDK4, CDK6, cyclin A and cyclin E, but these protein levels were unchanged (Fig. 6C).

\section{Discussion}

In this study, we aimed to unveil the global gene expression changes shortly after p16INK4A or p14ARF silencing. We revealed that the expressions of several genes are altered in the same manner in p16INK4A- and p14ARF-silenced HeLa cells. Among the identified genes, we focused on FBXL16, the function of which is so far unknown. We revealed that FBXL16 is a novel target of E2F1 and knockdown of FBXL16 increased the cell proliferation rate of HeLa cells.

The relation between carcinogenesis and $C D K N 2 A$ has been extensively studied $(1,2)$. As mouse models, p16Ink4aspecific and p19Arf-specific as well as double gene knockout mice have been produced and analyzed. In contrast to human cases, p19Arf-specific knockout mice exhibited a severer cancer-prone phenotype than p16Ink4a-specific knockout mice, though double-gene knockout mice exhibited the most malignant phenotype $(11,12)$. Therefore, revealing the molecular target just after p16INK4A or p14ARF inactivation using human cancer cells as an experimental model is an intriguing task. We selected HeLa cells, a cervical cancer cell line, because they express both p16INK4A and p14ARF proteins. As tumor suppressor proteins, p16INK4A and p14ARF are generally considered to be inactivated during the process of tumorigenesis $(1,2)$. However, some tumors possess upregulated levels of p16INK4A and p14ARF. For example, p16INK4A and p14ARF mRNAs are present at high 
A
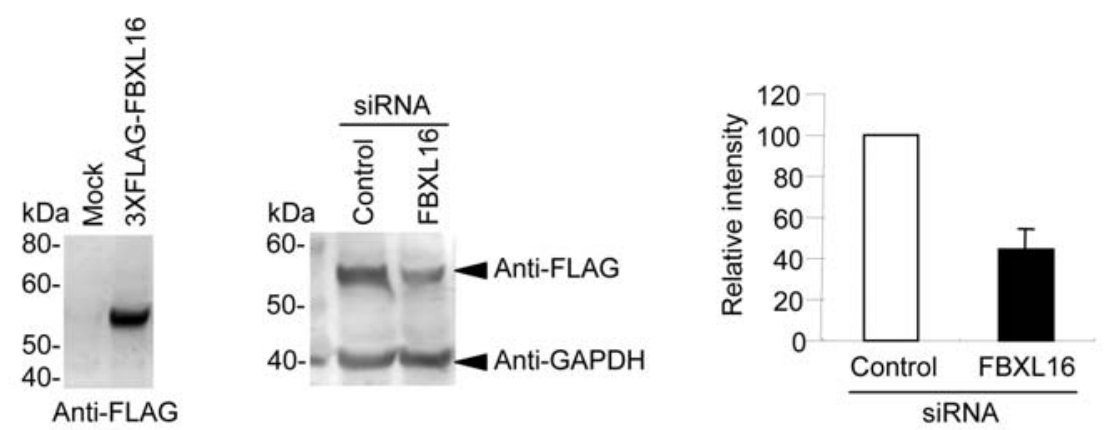

B

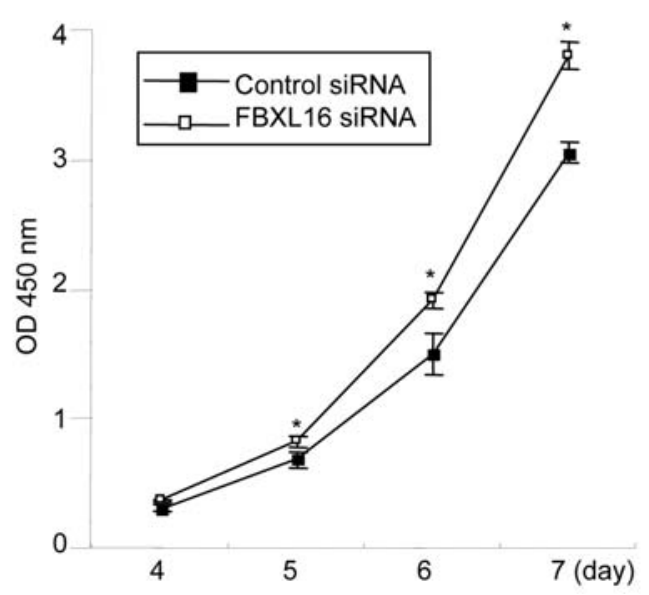

C

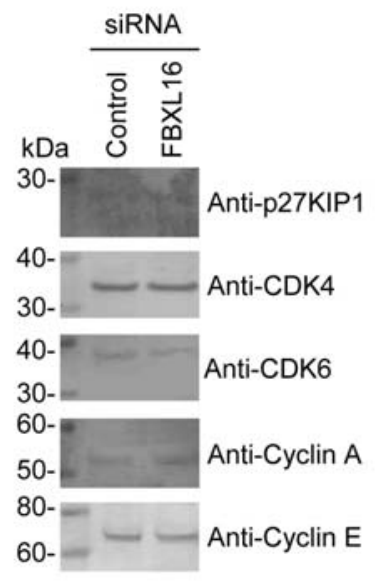

Figure 6. Effect of FBXL16 knockdown on cell proliferation. (A) RNAi-mediated knockdown of FBXL16 protein. Expression level of 3xFLAG-tagged FBXL16 in HeLa cells was detected using anti-FLAG (left panel). Mock indicates the empty vector p3xFLAG-CMV-10. FBXL16-specific or control siRNA were introduced into the cells on days 0 and 1 of RNAi protocol (see Materials and methods), and $24 \mathrm{~h}$ later FLAG-tagged FBXL16 expression vector was transfected into the siRNA pre-treated cells. Cell lysates were recovered on day 4 of RNAi protocol and the band was detected by anti-FLAG and antiGAPDH antibodies, respectively (middle panel). Molecular weight $(\mathrm{kDa})$ is shown on the left side of each panel. The efficiency of FBXL16-specific siRNA on FBXL16 protein level (right panel). The gel images were processed using Quantity One (Bio-Rad Laboratories). The band detected by anti-FLAG was normalized by the band detected by anti-GAPDH. Relative intensity was represented as the ratio of FLAG/GAPDH of control siRNA set as 100. Values are expressed as the means \pm S.D. $(\mathrm{n}=2)$. (B) WST-1 assay of FBXL16 knockdown cells during days 4-7 of RNAi protocol. The black and white boxes indicate the OD 450-nm value of the control and FBXL16 siRNA-treated cells, respectively. Values are expressed as the means \pm S.D. (n=3). ${ }^{*} \mathrm{P}<0.05$ (compared with control siRNA-treated cells), using a two-tailed Student t-test. (C) Western blot analysis of indicated cell cycle regulators. On day 3 of RNAi protocol, cell lysates of control and FBXL16 siRNA-treated cells were recovered. The molecular weight $(\mathrm{kDa})$ is shown on the left side of each panel.

levels in advanced stage and high-grade urothelial carcinomas (20). We do not have any mechanistic clues regarding the upregulation of p16INK4A and p14ARF in HeLa cells, but viral E6 and E7 oncogene products are potentially involved in such a process due to integration of HPV (human papillomavirus) genome. Indeed, viral E7 protein has known to induce increasingly expression of p16INK4A (21). But HeLa cells could be a beneficial model system for examining siRNA efficiency and global gene expression changes induced by the knockdown of p16INK4A and p14ARF. So far, siRNA-mediated p16INK4A and p14ARF suppression in cultured human cells has been established (22). The siRNAmediated knockdown of p16INK4A in late-passage normal human diploid fibroblasts resulted in a transitory escape from entry into replicative senescence (23). In contrast, the siRNAmediated knockdown of p16INK4A in human fibroblasts produced only a minimal lifespan extension that was terminated by senescence (24). Because our intention was to identify genes whose expressional levels were spontaneously affected and were relevant to acute p16INK4A loss as well as acute p14ARF loss, we preferred HeLa cells instead of primary fibroblasts. Similar to the present study, cervical cancer cell lines were also used in a previous study in which p16INK4A-silencing resulted in the upregulation of p53, $\mathrm{p} 21 \mathrm{WAF} 1 / \mathrm{CIP} 1$ and $\mathrm{pRb}$, and an increase in the apoptotic cell numbers induced by ultraviolet-irradiation and cisplatin treatment (16). However, we did not detect p53 upregulation in p16INK4A-silenced HeLa cells. Although HPV genome integration into the genome of HeLa cells compromises p53 function, p53 and its downstream target p21WAF1/CIP1 continue to function, as they can be induced by stress (25). The reason for this discrepancy has not yet been resolved.

A few crucial regulators upstream of the INK4A/ARF locus have been reported. For example, oncogenic HRAS can induce p16INK4A in primary human fibroblasts (26). In primary mouse embryonic fibroblasts deficient for Bmi1, the expression levels of p16Ink4a and p19Arf were markedly elevated and, conversely, Bmi1 overexpression downregulated the expressions of p16Ink4a and p19Arf (27). CDC6 overexpression has been shown to repress the expression levels of p16INK4 and p14ARF through the epigenetic modification of chromatin at the INK4/ARF locus (28). To 
see whether these upstream regulators can affect the INK4A/ $A R F$ locus in our assay system, we overexpressed oncogenic HRAS, Bmi-1 and CDC6, and checked the protein levels for p16INK4A, but no significant changes were observed. These results argue that our approach to the direct inactivation of p16INK4A and p14ARF could be a reasonable method of reproducing the situation during tumorigenesis and identifying the molecular networks that are modulated by the acute loss of both tumor suppressors.

No functional scrutiny on FBXL16 has been reported; however, genome-wide analysis of cell-cycle regulators by using RNAi has revealed that regulators of ubiquitin/ proteasome-mediated proteolysis including FBXO5, FBXL1, FBXL2, FBXL10 and FBXL16 had altered cell cycle progression in U2OS osteosarcoma cells (29). Among 1,152 genes $(4.7 \%$ of all genes studied) that altered cell cycle progression, downregulation of FBXL16 resulted in increased number of G2/M cells with large nuclei (29). Consistently, our result showed that knockdown of FBXL16 increased the cell proliferation rate of HeLa cells. FBXL16 has been identified as a member of the F-box protein family, which has a characteristic F-box motif, and it is thought to act as a protein-ubiquitin ligase component of SCF (Skp1, Cullins, F-box proteins) complexes (18). One needs to test whether FBXL16 is involved in ubiquitin-mediated degradation of certain cell cycle regulator as a component of SCF complexes. Currently we are attempting to establish FBXL16-inducible cell lines to see if there any differences in protein expression profile.

In summary, we have demonstrated that FBXL16 is a novel E2F1 target gene that is uniquely situated at a crossover in the downstream pathways of both p16INK4A and p14ARF. Further studies examining FBXL16 expression in various tumors and a functional analysis will shed light on whether FBXL16 might be potentially useful for the diagnosis and treatment of tumors.

\section{Acknowledgements}

We thank M. Kato, C. Lin and S. Takayanagi for technical assistance. This work was supported by Grant from the Daiwa Securities Health Foundation.

\section{References}

1. Sharpless NE and DePinho RA: The INK4A/ARF locus and its two gene products. Curr Opin Genet Dev 9: 22-30, 1999.

2. Sharpless NE: INK4a/ARF: a multifunctional tumor suppressor locus. Mutat Res 576: 22-38, 2005.

3. Lowe SW and Sherr CJ: Tumor suppression by Ink4a-Arf: progress and puzzles. Curr Opin Genet Dev 13: 77-83, 2003.

4. Sherr CJ: The INK4a/ARF network in tumour suppression. Nat Rev Mol Cell Biol 2: 731-737, 2001.

5. Elliott MJ, Dong YB, Yang H and McMasters KM: E2F-1 upregulates c-Myc and p14(ARF) and induces apoptosis in colon cancer cells. Clin Cancer Res 7: 3590-3597, 2001.

6. Mason SL, Loughran O and La Thangue NB: p14(ARF) regulates E2F activity. Oncogene 21: 4220-4230, 2002.

7. Gartel AL, Goufman E, Tevosian SG, Shih H, Yee AS and Tyner AL: Activation and repression of p21(WAF1/CIP1) transcription by RB binding proteins. Oncogene 17: 3463-3469, 1998.

8. Lee CW, Sørensen TS, Shikama N and La Thangue NB: Functional interplay between p53 and E2F through co-activator p300. Oncogene 16: 2695-2710, 1998.
9. Delavaine L and La Thangue NB: Control of E2F activity by p21Waf1/Cip1. Oncogene 18: 5381-5392, 1999.

10. Yang HL, Dong YB, Elliott MJ, Liu TJ, Atienza CJ, Stilwell A and McMasters KM: Adenovirus-mediated E2F-1 gene transfer inhibits MDM2 expression and efficiently induces apoptosis in MDM2-overexpressing tumor cells. Clin Cancer Res 5: 2242-2250, 1999.

11. Serrano M: The INK4a/ARF locus in murine tumorigenesis. Carcinogenesis 21: 865-869, 2000.

12. Berger JH and Bardeesy N: Modeling INK4/ARF tumor suppression in the mouse. Curr Mol Med 7: 63-75, 2007.

13. Box AH and Demetrick DJ: Cell cycle kinase inhibitor expression and hypoxia-induced cell cycle arrest in human cancer cell lines. Carcinogenesis 25: 2325-2335, 2004.

14. David-Pfeuty T and Nouvian-Dooghe Y: Human p14(Arf): an exquisite sensor of morphological changes and of short-lived perturbations in cell cycle and in nucleolar function. Oncogene 21: 6779-6790, 2002

15. Wang XQ, Gabrielli BG, Milligan A, Dickinson JL, Antalis TM and Ellem KA: Accumulation of p16CDKN2A in response to ultraviolet irradiation correlates with late $S-G(2)$-phase cell cycle delay. Cancer Res 56: 2510-2514, 1996.

16. Lau WM, Ho TH and Hui KM: p16INK4A-silencing augments DNA damage-induced apoptosis in cervical cancer cells. Oncogene 26: 6050-6060, 2007.

17. Krimpenfort P, Ijpenberg A, Song JY, van der Valk M, Nawijn M, Zevenhoven $J$ and Berns $A$ : p15Ink4b is a critical tumour suppressor in the absence of p16Ink4a. Nature 448: 943-946, 2007.

18. Jin J, Cardozo T, Lovering RC, Elledge SJ, Pagano M and Harper JW: Systematic analysis and nomenclature of mammalian F-box proteins. Genes Dev 18: 2573-2580, 2004.

19. Carrano AC, Eytan E, Hershko A and Pagano M: SKP2 is required for ubiquitin-mediated degradation of the CDK inhibitor p27. Nat Cell Biol 1: 193-199, 1999.

20. Le Frère-Belda MA, Gil Diez de Medina S, Daher A, Martin N, Albaud B, Heudes D, Abbou CC, Thiery JP, Zafrani ES, Radvanyi $\mathrm{F}$ and Chopin D: Profiles of the 2 INK4a gene products, p16 and p14ARF, in human reference urothelium and bladder carcinomas, according to $\mathrm{pRb}$ and $\mathrm{p} 53$ protein status. Hum Pathol 35: 817-824, 2004.

21. von Knebel Doeberitz M: New markers for cervical dysplasia to visualise the genomic chaos created by aberrant oncogenic papillomavirus infections. Eur J Cancer 38: 2229-2242, 2002.

22. Voorhoeve PM and Agami R: The tumor-suppressive functions of the human INK4A locus. Cancer Cell 4: 311-319, 2003.

23. Bond J, Jones C, Haughton M, DeMicco C, Kipling D and Wynford-Thomas D: Direct evidence from siRNA-directed "knock down" that p16(INK4a) is required for human fibroblast senescence and for limiting ras-induced epithelial cell proliferation. Exp Cell Res 292: 151-156, 2004.

24. Wei W, Herbig U, Wei S, Dutriaux A and Sedivy JM: Loss of retinoblastoma but not $\mathrm{p} 16$ function allows bypass of replicative senescence in human fibroblasts. EMBO Rep 4: 1061-1066, 2003.

25. Brown JP, Wei W and Sedivy JM: Bypass of senescence after disruption of p21CIP1/WAF1 gene in normal diploid human fibroblasts. Science 277: 831-834, 1997.

26. Serrano M, Lin AW, McCurrach ME, Beach D and Lowe SW: Oncogenic ras provokes premature cell senescence associated with accumulation of p53 and p16INK4a. Cell 88: 593-602, 1997.

27. Jacobs JJ, Kieboom K, Marino S, DePinho RA and van Lohuizen M: The oncogene and Polycomb-group gene bmi-1 regulates cell proliferation and senescence through the ink4a locus. Nature 397: 164-168, 1999.

28. Gonzalez S, Klatt P, Delgado S, Conde E, Lopez-Rios F, Sanchez-Cespedes M, Mendez J, Antequera F and Serrano M: Oncogenic activity of Cdc6 through repression of the INK4/ ARF locus. Nature 440: 702-706, 2006.

29. Mukherji M, Bell R, Supekova L, Wang Y, Orth AP, Batalov S, Miraglia L, Huesken D, Lange J, Martin C, Sahasrabudhe S, Reinhardt M, Natt F, Hall J, Mickanin C, Labow M, Chanda SK, Cho CY and Schultz PG: Genome-wide functional analysis of human cell-cycle regulators. Proc Natl Acad Sci USA 103: 14819-14824, 2006. 